\title{
Optimum Research of Control Law in HTY-X of Ejection Seat
}

\author{
${\text { Zhao } \text { Wei }^{1,} \text {, }}$, Wang Jingheng ${ }^{1}$, Meng Dongqi ${ }^{1}$ \\ ${ }^{1}$ Basic Flight Training Base, Avigation University of Air Force, Changchun, China 130022 \\ ayanh603@nenu.edu.cn
}

Key words: Ejection seat; Simulation; Maximum allowable velocity of parachute opening

\begin{abstract}
The effects of deceleration parachute of ejection seat on ejection trajectory were analyzed under low velocity condition. Through trial method and simulation, delay time of parachute opening was optimized in terms of maximum mobile load and maximum velocity. The results showed that the curve-optimized of delay time was positive correlated with maximum velocity of rescue parachute. Collectively, the curve -optimized of delay time may be used in the area of engineering instruction.
\end{abstract}

\section{Introduction}

As we all know, bi-state automatic control procedure for ejection seat is a much simpler model, which operates in terms of fixed time model at lower velocity and higher velocity. At medium velocity, the correlation degree of delay time on velocity is assessed by linear regression. Meanwhile, there are some shortcomings in the latter model. Delay time may be so short that parachute opening velocity should exceed maximum allowable value, and mobile load increases. In other cases, delay time may be so long that ejection process is postponed. In the field of lifesaving, it is generally accepted that the time of opening parachute should be decreased in order to save life. In low-altitude flight, improving the lifesaving performance of ejection seat should shorten the time of opening parachute rather than increase ejection power to make the parachute eject higher ${ }^{[1]}$. As for deceleration parachute, it plays a vital role in stabilizing the parachute and reducing the parachute velocity. We are not sure whether it is necessary to eject the parachute in low-altitude flight. Therefore no detail information is available on the research. The objectives of this study are to (1) optimize delay time of the parachute; (2) analysis the effects of deceleration parachute ejection on ejection trajectory.

\section{The operating principle of the automatic parachute opener with bi-state control}

Based on flight velocity of the airplane and curve of delay time, automatic parachute opener with bi-state control sets to work. Procedure in separation of deflagration system is triggered, accompanied with shoulder restraint strap - released, deceleration parachute-opened, and actuator cylinder-released. Then, deceleration parachute-released makes drogue gun set to work and eject rescue parachute. The pilot opens the lockers of leg restrain line and lap strap, and releases leg strap and lap strap. While rescue parachute is aired and filled with air, the pilot departs from ejection seat. In order to save his life, the pilot must be sure to descend steadily.

The automatic parachute opener with bi-state control is the mechanical-time control system. First of all, the airplane velocity is changed into pressure signal through air velocity head, which is changed into the mechanical displacement by the corrugated tube. Second, the mechanical displacement is turned into the guide block displacement. When airplane velocity is less than 450 
$\mathrm{km} \cdot \mathrm{h}^{-1}$, the guide block displacement is a constant value named $\mathrm{L}_{0}$. When airplane velocity varies from 450 to $850 \mathrm{~km} \cdot \mathrm{h}^{-1}$, the guide block displacement signed $\mathrm{L}$ is increased with increasing airplane velocity. When airplane velocity is more than $850 \mathrm{~km} \cdot \mathrm{h}^{-1}$, the guide block displacement is also changed into a constant value named $\mathrm{L}_{\max }$. As the airplane flies, the guide block displacement signed L is changed constantly with the variation of airplane velocity. Once ejection seat is ejected from the airplane, the guide block and the displacement signed $\mathrm{L}$ are all fixed. Therefore, airplane velocity and the relative $\mathrm{L}$ value are stored. As soon as ejection seat leaves the airplane, the clock mechanism of parachute opener will begin to work, the rotation angle of the eccentric wheel is changed into the displacement (signed S) through the transmission system. When the $\mathrm{S}$ value is equal to the $\mathrm{L}$ value, the clock mechanism is limited. This mechanism ensures that the time of parachute opening might be changed with the variation of airplane velocity. In fact, the ejection is a kind of complex and continuous process, and always is affected by several factors. In order to calculate conveniently, the ejection process is divided into several stages, including extravehicular stage, free flight stage, flare-out stage of rescue parachute, and inflating stage of rescue parachute. Based on the movement characters of different stages, the mathematical models are developed ${ }^{[2]}$.

\section{Partition of the delay time for the ejection parachute}

At present, available exact information regarding delay time for ejection parachute is limited. Because the ejection process is always affected by several uncertain factors, it is difficult for the traditional linear or nonlinear optimization method to popularize in ejection lifesaving area. Although objective function is confirmed, ejection lifesaving is composed of different stages. It is difficult to describe in the terms of a unified objective function. The paper realizes the delay time-optimized for ejection parachute by the way of calculation. On the basis of different velocity -chosen and different delay time of ejection parachute, we should ensure if calculating the velocity and the mobile load of parachute opening meets requirements. According to delay time of FAST program controller and the control curve for delay time (QKS-14), some certain velocity points were chosen as the simulation targets in the above velocity range. Then, we tested whether parachute opening velocity of such delay time was higher than maximum allowable velocity of parachute opening $\left(500 \mathrm{~km} \cdot \mathrm{h}^{-1}\right)$. Based on velocity points-chosen, delay time of ejection parachute was regulated until maximum allowable velocity of parachute opening and maximum mobile load of parachute opening were achieved.

Through the simulation calculation, delay time of ejection parachute in medium and higher ranges was divided four scopes, i.e., $450-650 \mathrm{~km} \cdot \mathrm{h}^{-1}, 650-700 \mathrm{~km} \cdot \mathrm{h}^{-1}, 700-750 \mathrm{~km} \cdot \mathrm{h}^{-1}, 750$ $850 \mathrm{~km} \cdot \mathrm{h}^{-1}$. The segmented linear time mode lied in the velocity-points choice following these steps. When velocity-point was $450,650,700,750,850 \mathrm{~km} \cdot \mathrm{h}^{-1}$, the delay time was $0.7,1.2,1.6$, 1.7, $1.9 \mathrm{~s}$, respectively. Based on the lowest safety life-saving height, the ejection life-saving time, maximum mobile load of parachute opening, and parachute opening velocity, delay time was regulated properly. In the paper, the mobile load was determined by using the formula as follow ${ }^{[3]}$ :

$$
\begin{aligned}
& \mathrm{F}_{\mathrm{k}}=\left(m_{s}+m_{f}\right) \frac{d v_{m}}{d t}+V_{m} \frac{d m_{f}}{d t}+Q_{s}+m_{s} g \sin \theta_{w} \\
& N_{k}=\frac{F_{k}}{m_{w} g}
\end{aligned}
$$

Where $F_{k}(N)$ is acting force of parachute opening, $m_{s}(\mathrm{~kg})$ is parachute weight, $\theta_{w}(\mathrm{rad})$ is included angle between motion trail of parachute and horizontal plane, $m_{f}(\mathrm{~kg})$ is associated mass of air, $\mathrm{Q}_{\mathrm{s}}$ 
$(N)$ is air resistance of parachute, $V_{m}(\mathrm{~m} / \mathrm{s})$ is velocity of human and parachute, $N_{k}$ is mobile load of parachute opening, $m_{w}(\mathrm{~kg})$ is the crew mass.

\section{Simulating the delay time-optimized for the ejection parachute}

During the simulation, the fifth percentile was defined as the crew standard, the eccentric distance was $0.04 \mathrm{~mm}$, simulation attitude was showed in leveled state. Because it was ejected in leveled state, ejection seat should be able to meet the requirement of life-saving height. Comparatively, maximum mobile load of parachute opening and maximum allowable velocity of parachute opening were the main parameters in the simulation.

Table 1 showed the parameters comparison between the interval endpoints of different velocity and the velocity point of delay time in parachute opening. The results showed that velocity point in delay time curve basically met the requirement of maximum allowable velocity of parachute opening. Although maximum mobile load of parachute opening followed up with the requested the national military standard in delay time curve of automatic parachute opener, velocity point of parachute opening exceeded maximum allowable velocity.

In order to verify whether velocity point in delay time curve basically should meet the requirement of maximum allowable velocity and maximum mobile load of parachute opening or not, velocity point in the different scope was randomly chosen and compared. The results in Table 2 showed that all the velocity points were consistent with the maximum allowable velocity except for the velocity of $800 \mathrm{~km} \cdot \mathrm{h}^{-1}$ in which velocity of parachute opening was greater than $500 \mathrm{~km} \cdot \mathrm{h}^{-1}$.

Table1 Comparative parameters in interval endpoints of different velocity

\begin{tabular}{|c|c|c|c|c|c|c|}
\hline \multirow{2}{*}{ Velocity $\left[\mathrm{km} \cdot \mathrm{h}^{-1}\right]$} & \multicolumn{3}{|c|}{ Simulation } & \multicolumn{3}{|c|}{ HTY-X } \\
\hline & Delay time[s] & $V_{\mathrm{k}}\left[\mathrm{km} \cdot \mathrm{h}^{-1}\right]$ & $N_{\text {kmax }}[\mathrm{g}]$ & Delay time[s] & $V_{\mathrm{k}}\left[\mathrm{km} \cdot \mathrm{h}^{-1}\right]$ & $N_{\text {kmax }}[\mathrm{g}]$ \\
\hline 650 & 1.20 & 451 & $10-11$ & 1.25 & 440 & $9-10$ \\
\hline 700 & 1.60 & 488.2 & $13-14$ & 1.39 & 518.2 & $14-17$ \\
\hline 750 & 1.70 & 489.7 & $12-14$ & 1.53 & 517.7 & $14-17$ \\
\hline 850 & 1.90 & 485.7 & $12-13$ & 1.80 & 432.1 & $14-16$ \\
\hline \multicolumn{7}{|c|}{ Table2 Comparative parameters in any velocity point } \\
\hline \multirow{2}{*}{ Velocity $\left[\mathrm{km} \cdot \mathrm{h}^{-1}\right]$} & \multicolumn{3}{|c|}{ Simulation } & \multicolumn{3}{|c|}{ HTY-X } \\
\hline & Delay time[s] & $V_{\mathrm{k}}\left[\mathrm{km} \cdot \mathrm{h}^{-1}\right]$ & $N_{\text {kmax }}[\mathrm{g}]$ & Delay time[s] & $V_{\mathrm{k}}\left[\mathrm{km} \cdot \mathrm{h}^{-1}\right]$ & $N_{\text {kmax }}[\mathrm{g}]$ \\
\hline 580 & 1.03 & 458.4 & $10-11$ & 1.06 & 448.1 & $9-10$ \\
\hline 680 & 1.44 & 370.1 & $5-6$ & 1.33 & 424.4 & $8-9$ \\
\hline 730 & 1.60 & 489.4 & $12-14$ & 1.47 & 521.4 & $14-17$ \\
\hline 800 & 1.80 & 511.4 & $13-16$ & 1.66 & 537.3 & $16-18$ \\
\hline
\end{tabular}

$* V_{\mathrm{k}}$ : Velocity of parachute opening

\section{The effect of the deceleration parachute ejection on the ejection trajectory}

The stabilization mode of deceleration parachute, which is adopted in HTY-X ejection seat, plays in role in stabilizing and decelerating the ejection seat. The deceleration parachute was triggered, and ejected 0.4 second later ${ }^{[4]}$. Therefore no detail information is available on the research of parachute ejection in low-altitude flight. Through simulating ejection conditions, such as parallel track, dive, and roll, the effects of deceleration parachute ejection on safe altitude are analyzed ${ }^{[5]}$.

When deceleration parachute can not be sent out, there are two ways for rescue parachute. One way is that rescue parachute will be sent out immediately once ejection seat leaves the airplane, the other way is that the ejection of rescue parachute will be delayed according to delay time set by the automatic parachute opener. During the simulation, the 95th percentile is defined as the crew standard, the eccentric distance is $0.04 \mathrm{~mm}$, the ejection exit time 0.01 second, working time for 
rocket pack is 0.25 second, ejection velocity is $120 \mathrm{~km} \cdot \mathrm{h}^{-1}$.

Under the above motioned ejection condition, the simulation curve of ejection trajectory was shown in Figure 1-3. In lower velocity condition, ejection trajectory was less affected by the ejection of the deceleration parachute. The two curves were almost overlapped each other. However, rescue parachute, ejected immediately, should improve ejection trajectory after seat ejection.

Under the ejection condition of $120 \mathrm{~km} \cdot \mathrm{h}^{-1}$ velocity and $60^{\circ}$ roll angle, the variations of pitching angle, yaw angle and roll angle were shown in Figure 4-5. From the results, rescue parachute could not play the role in stabilizing ejection seat under low velocity condition. Before ejecting parachute, the changes of the two curves were consistent with each other. Owing to delaying the time of rescue parachute ejection, the mode of ejecting deceleration parachute further improved the ejection seat position. In the certain condition, this mode has no beneficial effects on life saving. In total, reducing ejection time of rescue parachute has benefit in improving lifesaving performance under low velocity condition. The result is in accord to the mode reported by Martin-Baker Aircraft Company Limited.

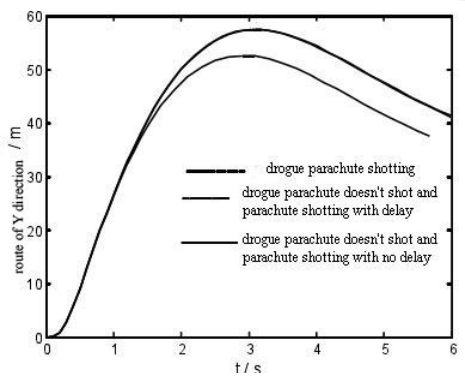

Fig.1: Ejection trajectory of ejection seat in flight level

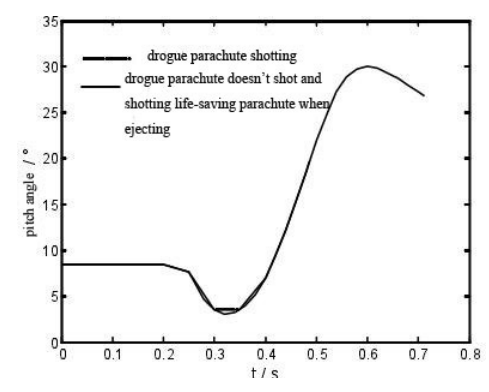

Fig. 4: Variation of pitching angle

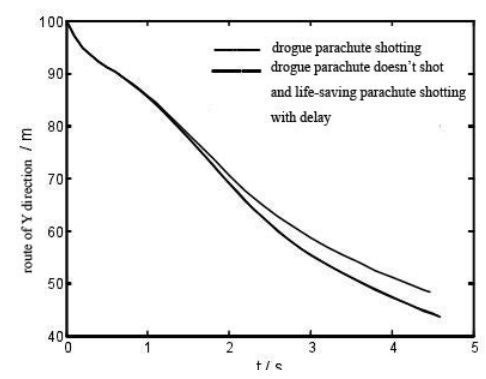

Fig.2: Ejection trajectory of ejection seat in $60^{\circ}$ dive angle

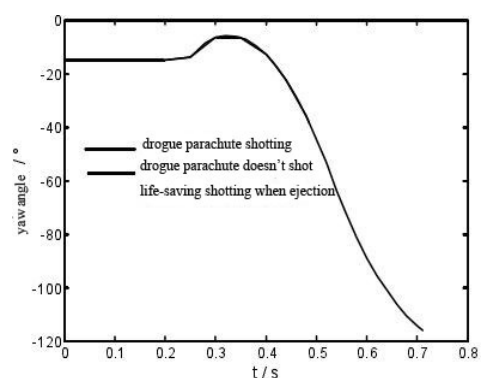

Fig. 5: Variation of yaw angel

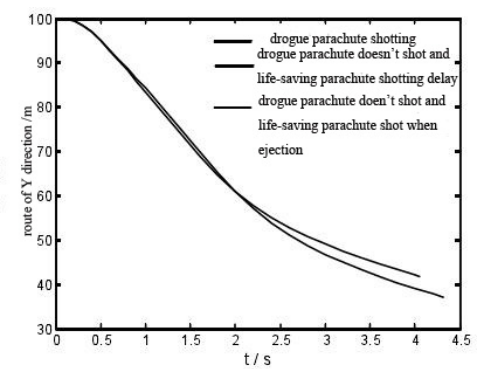

Fig.3: Ejection trajectory of ejection seat in $120^{\circ}$ roll angle

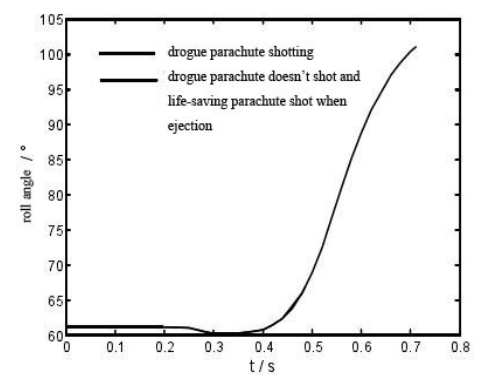

Fig. 6: Variation of roll angle

\section{Conclusion}

Through simulation, the paper optimized delay time curve of automatic parachute opener with bi-state control. The results showed that curve-optimized was positive correlated with maximum velocity of rescue parachute. In addition, the effects of deceleration parachute ejection on ejection trajectory and position changes of human- ejection seat were analyzed by simulation. The results indicated that ejection trajectory was less affected by deceleration parachute ejection in lower velocity condition. In such case, it did not take a role in stabilizing the ejection seat. However, the lifesaving performance is improved through reducing ejection time of the rescue parachute.

\section{Reference}

[1] H.B. Zhang. Aircraft safety and rescue. Beijing: Beijing University of Aeronautics and Astronautics, (1990).

[2] M. Deren. Biodynamic modeling and simulation of the ejection seat/occupant system. United 
States Air Force Research Laboratory, (2000).

[3] A. L. Obergefell, I. Kalepsa. Simulation of body motion during aircraft ejection. Mathematical and Computer Modelling, 11: 436-439, (1988).

[4] R.B. Calkins, G.M. Roe. ACES II Ejection Seat Cooperative Modification Project - Improved Stability Subsystem. Proceeding of the 39th SAFE Annual Symposium. America: SAFE Symposium, 221-231, (2003).

[5] M. Etson. Object orientated ejection seat mode. AIAA-1997-31357, (1997). 